\title{
Plain Geometry, a Novel Approach to Increase Solar Panel Efficiency
}

\author{
Parisa Khashayar ${ }^{1}$ and Eric Trumbauer ${ }^{1}$ \\ ${ }^{1}$ St. Margaret's Episcopal School, San Juan Capistrano, CA, USA \\ DOI: https://doi.org/10.47611/jsrhs.v10i2.1452
}

\section{$\underline{\text { ABSTRACT }}$}

Traditional flat solar panels with solar tracking and cooling systems are known to be very inefficient, expensive, and difficult to maintain. Additionally, their large footprints make it difficult to install in largely populated areas. This paper examines the advantages of using 3D solar panels in pyramid shapes over the conventional flat panels.

\section{Introduction}

Harvesting solar power into usable electricity is one of the most sought after and practical solution for renewable energy. Geometry and angles at which the sun's rays hit the panel play a direct role in a solar panel's capacity to convert the sun's energy to electricity. In order to capture the sun's energy, solar rays need to hit the panels directly on their surface, yet excessive heat may result in loss of efficiency in conversion. Conventional solutions such as solar tracking and cooling systems make the solar panel more expensive. Additionally, solar panels often require large areas for installation which make them even less practical in urban areas. This experiment proposes a three-dimensional shape in place of traditional flat panels, in order to tackle such issues as cost and footprint, while achieving maximum efficiency.

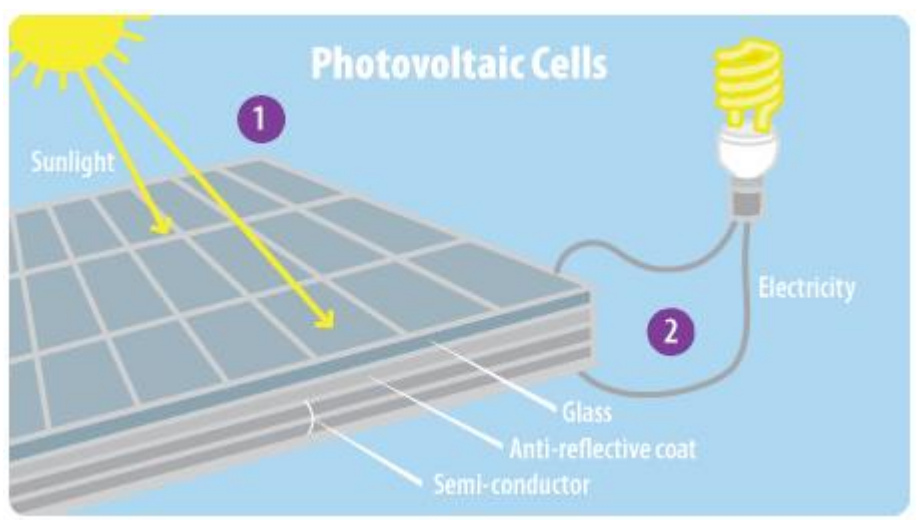

Figure 1: Solar panel technology. (Source: EPA website)

\section{Definition of terms}

\section{PV or Photo Voltaic}

process through which sunlight's energy converts to electricity in solar panels using semiconductor material. 


\section{Solar energy}

Sunlight is made of small particles called photons which radiate from the sun. When Photons hit the solar panel, they transfer their energy to the material that the solar panel is made out of.

\section{Semiconductor}

A group of elements from periodic table that has some properties of metals. Silicon is one of these elements.

\section{Atom}

An atom of any element in periodic table is made out of a proton and neutron and electron. Electrons, which are negatively charged, orbit around positively charged protons.

\section{Energy conversion}

Energy harvested from sun can be converted to heat or electricity.

\section{Light reflection}

When light hits a smooth surface, the light beam can bounce off the surface based on the material and color of the surface. The light bounces off the surface with the same angel it hits it. The un-reflected portion of the sun light (photons) is absorbed by the surface of the solar panel.

\section{Efficiency}

refers to the portion of energy in the form of sunlight that can be converted via photovoltaic cells into electricity. It is basically a ratio of output energy of the solar panel to input energy from the sun. It depends on intensity and wavelength of the sunlight as well as temperature of the solar panel.

\section{The need to harvest solar energy}

Global warming has become one of the most pressing issues in modern society. One of the compelling solutions is to eliminate the need for use of fossil fuels that pollute the Earth. Solar energy is an alternative and ultimate clean source for renewable clean energy. However, with all the new advancements we are still far from building efficient solar panels. Most panels can only convert $15 \%-20 \%$ of the daily energy they collect. Abundance of a more efficient solar panels would lead to larger employments of this technology by making it more accessible and less costly than traditional polluting fuels.

\section{How does a solar panel work?}

PV (Photovoltaic) Solar Cell uses semiconductors to convert sunlight directly to electricity. All solar cells require a light absorbing material, semiconductor to absorb photons and generate free electrons in the atoms by raising their energy level. Photons from the sun's rays excite electrons in solar cells and generate current (flow of electron) in each cell. A solar panel is comprised of many linked solar cells. The efficiency of a solar panel is the percentage of generated electricity to input solar energy. This information would determine the size of a solar panel is needed to produce a certain amount of energy. The higher the efficiency, the less surface area is required for the solar panels. Figure below shows how the energy of sun in the form of photon is absorbed by electrons on the surface of the solar panel. This energy knocks the electron free from its atom and therefore produces flow of current from one atom to another. 


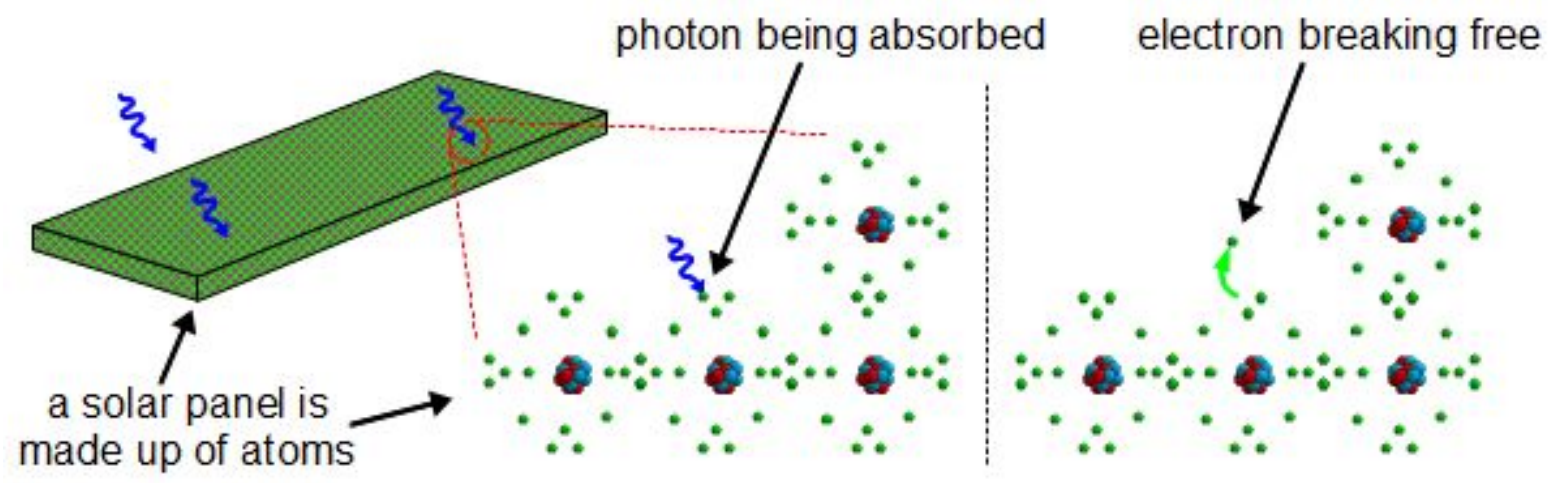

Figure 2: Energy from sun's rays breaking away electrons in semiconductors. (Source EPA website)

\section{Solar tracking}

Solar trackers are electromechanical devices that track sun's movement from east to west. They rotate the solar panel towards the sun to maximize the collection of sun's rays. The use of solar trackers can increase electricity production by around one fourth; however, they require some of that generated energy to operate their electronics and mechanical systems. The installation and operation of tracking system is often complicated and costly. Figure 3 illustrate how typical trackers move the solar panel.

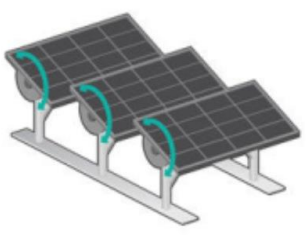

Single-Axis Tracker Illustration

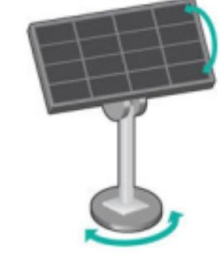

Dual-Axis Tracker Illustration

Figure 3: typical solar trackers can move the panel in one or two axis. (Source: exactsolar website)

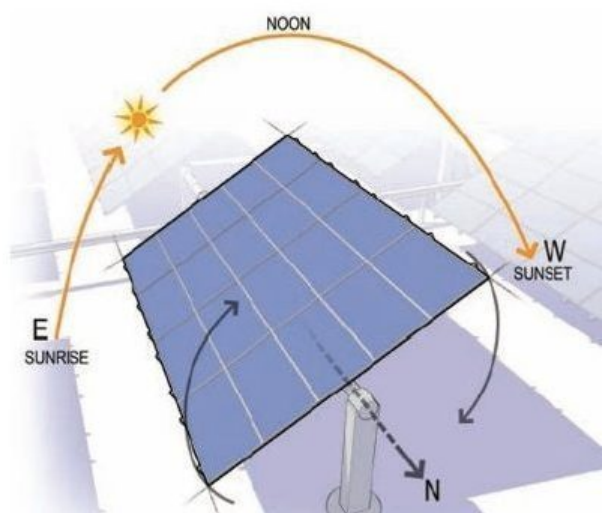

Figure 4: Rotation of solar panel to maximize the caption of sun's rays. (Source: exactsolar website) 


\section{Two major problems in current solar panel designs are efficiency and overheating.}

\section{Not capturing all available photons from the sunlight}

In order to absorb most of the photons from sunlight, the reflection of the sunbeam from the surface of the solar panel should be minimized. New panels use Anti-Reflection, (AR) coating which are expensive chemical material. Furthermore, the effectiveness of this material depends on the wavelength of sun rays and their angle with respect to the panel. Addition of high-quality AR coatings could increase the overall cost of solar panels by about $15 \%$. Also, many solar panels use a sun tracking system to follow the sun light and increase the chance of capturing maximum photons possible. Sun tracking systems are complicated, expensive and require additional energy to run, which could take away from the energy produced by the sun.

\section{Heat}

Solar panels are made out of semiconductor material that can behave differently at high temperatures. Extreme heat can reduce the semiconductor's capability to produce free electrons and therefore generating electricity. Traditional approach to heating problem is utilizing a cooling system using water, but addition of these systems and the extra energy it takes to run the cooling system also lowers the efficiency of the solar panel.

\section{The Plan}

\section{Question}

The three most popular approaches to increase efficiency of solar panels, water cooling, sun tracking and AR Coating are proven to be expensive and take away from overall energy produced by solar panels. Simple laws of physics and geometry can improve the efficiency of the solar cells as much as these traditional solutions but with a fraction of the cost or required area.

\section{Developing a solution}

The objective of this paper is to build a solar panel using an inside out and upside down pyramid, as a low cost solution to solve the classic solar panel problems such as overheating, space, and efficiency. This upside down pyramid can be more efficient than a generic flat plan and as efficient as solar panel with sun tracking systems, at the fraction of the cost and smaller footprint.
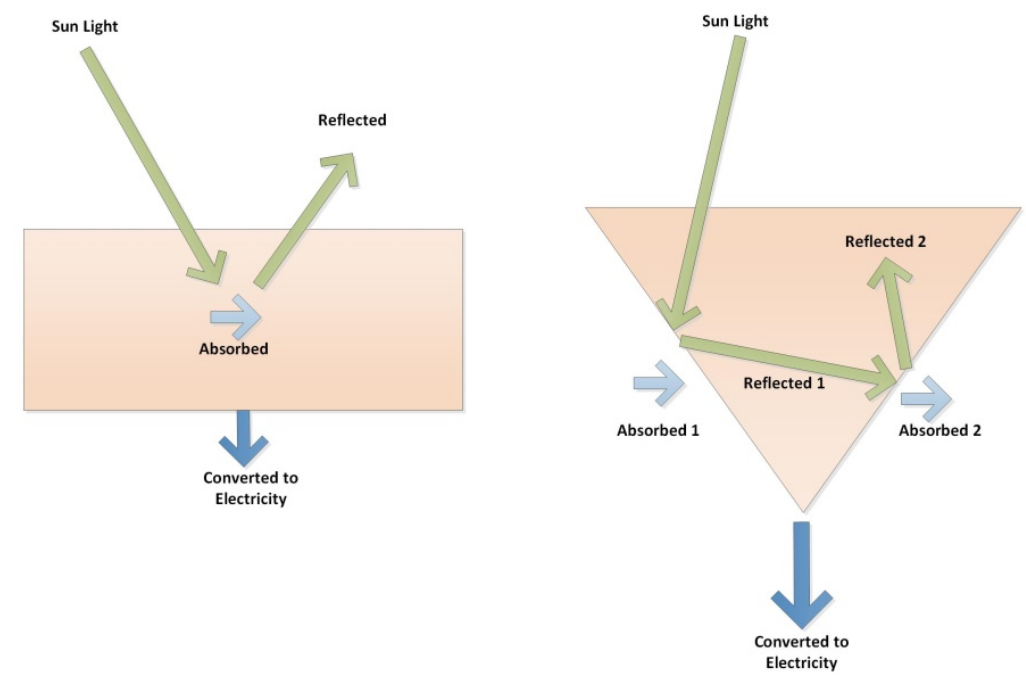

Figure 5: Absorption and reflection of sun's rays from a surface of a flat solar panel vs. pyramid solar panel 
Ultimately the pyramid shape of the solar panel reduces the total area used by the panel and improves the cooling of the panel by eliminating the requirement of facing the sun at all time.

According to the law of reflection, the angle of the beam hitting a reflective surface (angle of incident) is equal to the angle from which the beam is reflected back (angle of reflection). This experience uses this law to capture the reflected sunbeam and its photons and therefore avoid wasting any photons radiated from the reflected beam.

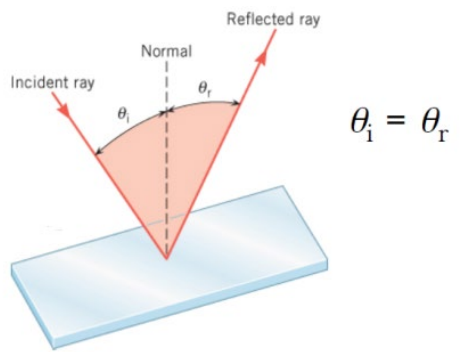

Figure 6: Law of reflection

With traditional flat solar panel, once the sun rays hit the panel, only small percentage of rays gets absorbed by it and the rest get reflected from it. An upside down pyramid uses multiple surfaces to catch the reflected rays from other panels, therefore increases the absorption rate of solar panel. Higher absorption rate means higher efficiency for solar panel. Figure 7 shows the concept of reflection from solar panel surface based on the angle of incidence (the angle from which the sun rays hit the panel). The closest the angle gets to 90 degrees, the higher the percentage of reflected sun rays from the panel.

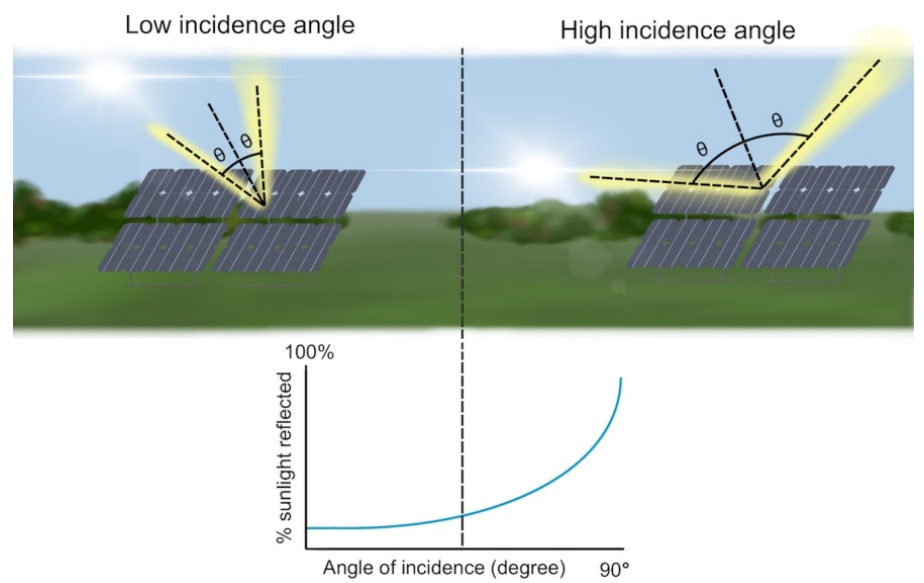

Figure 7: Sunlight reflection percentage based on the angle of incidence. (Source: forgesolar website)

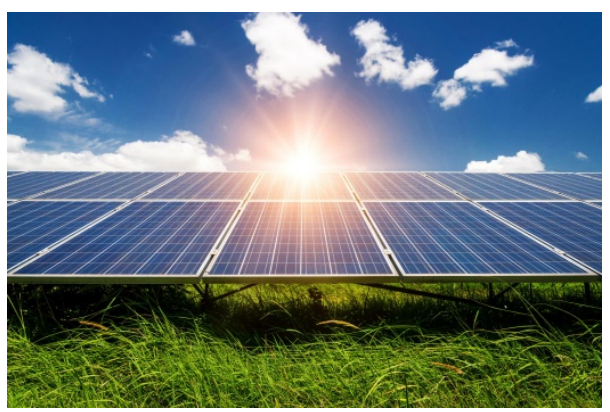

Figure 8: Sunlight reflecting back from the solar panel. 


\section{Calculating efficiency}

Efficiency of a PV solar panel is define, measured or calculated in few different ways. This paper will discuss the two most popular ways and the reason to choose one over the other.

1. Industrial: The solar panel manufactures use this formula to determine the efficiency of their solar panel and use this information to determine how many panels to be installed on a rooftop to generate the desire power.

The general formula is shown here:

$$
\begin{gathered}
n=\frac{\text { VocIscFF }}{P i n} \\
\text { Pmax }=\text { VocIscFF }
\end{gathered}
$$

n (efficiency) is calculated by maximum power output of solar panel divided by input power of sun light.

Voc is an open circuit voltage (volts). Maximum voltage the solar panel can produce without any load.

Isc is short circuit current (amps). It is the current of solar cell when voltage across the cell is zero.

FF is the fill factor which is a parameter of the semiconductor material. FF is the product pf Voc and Isc where maximum power is made. $\mathbf{F F}=\mathbf{V o c} \times \mathbf{I s c}$

Pin is the input power of sunlight that is calculated with the formula $\quad$ Pin $=\mathbf{E}^{*} \mathbf{A c}$

$\mathbf{E}$ is the incident radiation flux (W/m2). This information can be obtained from a general TCS table and is fixed in any given part of the world. This number is $1000 \mathrm{~W} / \mathrm{m} 2$ in USA.

Ac is the area of collector (m2)

\section{Science project:}

For the purpose of this project, a simple formula is used:

\% efficiency $=($ Power output of PV cell / Power input of sun $) X 100$

\section{Power output of cell per unit area:}

$$
\begin{gathered}
\text { Po }=\frac{\text { (Voltage X Current) }}{\text { Area }}=\frac{\text { Watts }}{\mathrm{cm} 2} \\
\text { Po }=\frac{\text { Watts }}{\mathrm{cm} 2}=10000 \times \frac{\text { Watts }}{\mathrm{m} 2}
\end{gathered}
$$

\section{Power input of the sun per unit area}

The amount of sun's energy that reaches the Earth's atmosphere is a known as Solar Constant and it is about 1350 Watts $/ \mathrm{m} 2$. It varies slightly depends are the location of solar cells on Earth and time of the year. The estimated value for the Sun's energy that reaches to the Earth's surface in a sunny day according to a time of the year is:

Sunny summer day: 1000 Watts/m2 
Sunny autumn or spring day: 900 Watts $/ \mathrm{m} 2$

Sunny winter day: 700 Watts/m2

This experiment suggests using the second method of calculating the efficiency. The formula of Po/Pin, which is the foundation for calculation of efficiency for any powered machine, is accurate and simple. This formula can easily determine the efficiency of this solar panel design in a meaningful way.

\section{Test procedure}

A controlled group should be created to test the efficiency of the design against that control group. The control group is a set of solar panels lying flat on the ground. Three sets of variables can be introduced and tested.

1. Solar panel equipped with solar tracking.

2. A regular 3-dimensional pyramid with solar panels mounted on the outside walls.

3. An upside-down pyramid with solar panels installed in the inside wall.

Same number of solar panels will be grouped and installed for each case to be tested under the same condition. For this experiment, a solar panel with flexible material is preferred. All four solar panel designs -three variables and one controlled set- should be placed in the same area under the sun. Then the output power of each set should be measured at three different time of the day -morning, noon, afternoon. The power output can be measure using volt/current meter. For the solar tracking panels, a simple motor from Lego Mindstorms can be utilized as a solar tracking unit to rotate the panels to a certain angle at a given time to simulate tracking the sun at different time of the day. The temperature on the surface of each set of shapes should also be measured and recorded to determine which one is the coolest.

\section{Conclusion}

The intent of this paper is to promote an out of the box approach to existing solar panel design. This research introduces a possible solution to reduce cost while improving efficiency of a solar panel by simply considering more effective geometry,"3D shapes", than traditional flat panels. Simple Physics can replace costly electronics and hardware that is needed to operate traditional solar tracking and cooling systems. Furthermore it abolishes the need to use any of the generated solar energy to operate these systems. Smaller installation area makes solar energy more attractive in urban areas, while elimination of costly electronics and hardware makes this solution more feasible in remote areas. There will be a less frequent need for maintenance and operation of these panels.

Utilizing a pyramid shape, the system can capture the sun's ray from any angle and location in the sky eliminating the need for expensive electronic solar tracking. Furthermore, carefully measured angles of the pyramid walls, facilitates the reflection of the rays back to the adjacent walls of the pyramid. Multiple reflection of the same sun ray into several panels will results in utilizing the rays more efficiently. This way the total sum of converted energy from a refracted ray would be more than the energy of just single ray absorbed by the conventional flat/single angle panels. Additionally, the pyramid shape helps cooling down the panels more efficiently than flat panels. Finally, typically, Solar panels lay flat on the ground or parallel to rooftops, this configuration leads to low efficiency and subsequently increase in number of solar panels used to generate the required energy. Smart use of geometry could reduce footprint and size of solar panels and make them more attractive for urban use. The exact percent increase in efficiency in this experiment is unknown as this experiment is undergoing additional research. 


\section{Acknowledgments}

I would like to thank Dr. Trumbauer for helping me with this project.

\section{References}

1. J. Rizk and Y. Chaiko, July 2008, "Solar tracking system, more efficient use of solar panels", world academy and science, engineering and technology. https://www.researchgate.net/publication/242528818 Solar Tracking System More Efficient Use of Solar Panels

2. Bhubaneswari Parida, S. Iniyan, A review of solar photovoltaic technologies, April 2011, https://www.sciencedirect.com/sciencelarticle/pii/S1364032110004016

3. Kevin Vu, How does solar cell work? Jan $9^{\text {th }} 2009$, https://www.physics.org/article-questions.aspid=51

Electrical Engineering, How to measure the maximum power of a solar panel, June 2012, https://electronics.stackexchange.com/questions/6489/how-to-measure-the-maximum-power-of-a-solarpanel

4. Charles R. Landau, Optimum tilt of solar panels, May 2015, https://www.solarpaneltilt.com/

5. Nancy S. Giges, Making solar panels more efficient, April 2014, https://www.asme.org/topics-resources/content/making-solar-panels-more-efficient

6. Fritz Albregstsen, The law of Reflection, Science learning center, June 2008 https://www.pveducation.org/pvcdrom/solar-cell-operation/solar-cell-efficiency

7. https://www.uio.no/studier/emner/matnat/ifi/INF-GEO4310/h09/undervisningsmateriale/imaging-kap2.pdf 